\title{
Left-handed properties of erbium-doped crystals
}

\author{
Quentin Thommen and Paul Mandel \\ Optique Nonlinéaire Théorique, Université Libre de Bruxelles, Campus Plaine CP 231, 1050 Bruxelles, Belgium
}

Received January 24, 2006; revised March 19, 2006; posted March 27, 2006 (Doc. ID 67461)

We show that lanthanide-doped crystals can be made left handed for a finite spectral range. The electronic transitions in the dopant ions are both dipolar electric and dipolar magnetic allowed. This enables tuning the permittivity and the permeability at the same frequency. The analysis focuses on erbium-doped crystals where left-handed properties are predicted inside an experimentally reachable domain of parameters. (C) 2006 Optical Society of America

OCIS codes: $160.5690,230.1150,020.1670$.

Media having negative values of the real parts of their permittivity and permeability (called lefthanded media) are promising materials for optical devices. They have recently attracted significant attention since it has been discovered that a slab of lefthanded material is able, for instance, to focus light into a spot much smaller than the wavelength, realizing a "perfect" lens. ${ }^{1,2}$ Experimentally, the first lefthanded properties were obtained in the microwave domain with a composite medium made of a periodic alternation of split ring resonators and continuous wires $^{3}$ and more recently in the near-infrared domain with a doubly periodic array of pairs of parallel nanorods. ${ }^{4}$

In this Letter left handedness in lanthanide-doped crystals is analyzed. In our model an electric transition and a magnetic transition play the role of an electric resonator and a magnetic resonator, respectively. Explicit calculations for an erbium-doped crystal predict the occurrence of a left-handed domain for a probe field oscillating at approximately $1.54 \mu \mathrm{m}$. Contrary to previous studies on atomic gases, ${ }^{5,6}$ no strong electromagnetic fields are required.

Let a medium interact with a weak probe electromagnetic beam oscillating at frequency $\omega$ and described by the electric field $\boldsymbol{E}$, the magnetic field $\boldsymbol{B}$, and the wave vector $\boldsymbol{k}$. During the interaction, the medium develops a macroscopic polarization $\boldsymbol{P}$ and a macroscopic magnetization $\boldsymbol{M}$. These are the mean values of the atomic electric dipoles and the atomic magnetic dipoles. We assume a linear response $\boldsymbol{P}$ $=\varepsilon_{0} \alpha_{e} \boldsymbol{E}$ and $\mu_{0} \boldsymbol{M}=\alpha_{m} \boldsymbol{B}$, where $\alpha_{e}$ and $\alpha_{m}$ are the polarizability and the magnetizability, respectively. ${ }^{7} \mathrm{~A}$ magnetizability and a polarizability of order unity and at the same frequency are required to obtain a domain of parameter with negative real parts of the relative permeability $\mu_{r}=\left(1-\alpha_{m}\right)^{-1}$ and of the relative permittivity $\varepsilon_{r}=1+\alpha_{e}$. An electronic transition involving an electric (magnetic) dipole tunes the permittivity (permeability) in the same way as an electric (magnetic) resonator having the transition frequency among its eigenfrequencies. Therefore a medium having a nearly degenerate electric dipolar transition and magnetic dipolar transition is a good candidate to realize a left-handed material around the transition frequencies. This can be found in lanthanidedoped crystals.
The lanthanides correspond to the 14 elements with an unfilled $4 f$ subshell. According to the Klechkowski rules, the $5 s$ and $5 p$ subshells are populated before the $4 f$ subshell. Thus the inner unfilled $4 f$ subshell is compressed and shielded by the outer filled $5 s^{2}$ and $5 p^{6}$ subshells. Therefore the $4 f$ electrons play a small role in the chemical bounding and behave as in a free ion. Spectroscopic properties of lanthanide ions embedded in a host lattice are well described by the crystal field theory. ${ }^{8}$ In this picture lanthanide ions are considered as free ions in a strong electric field created by the surrounding ions belonging to the host lattice. For the lanthanide ions, the interaction of an electron with the crystal field is much weaker than the Colombic or the spin-orbit interactions. Therefore the total angular momentum $J$ still remains a good quantum number. A first effect of the crystal field is to remove the magnetic degeneracy by the Stark effect. Moreover, the crystal field induces Stark mixing between the opposite-parity configurations if the lanthanide ions are placed in noncentrally symmetric sites of the host lattice. In this case, the transitions between the states of the $4 f$ configuration become electric dipole allowed while they are forbidden in a free ion. ${ }^{9}$ These properties have been extensively studied to realize laser crystals. ${ }^{10}$ The selection rules concerning the magnetic quantum number for the electric dipolar transition induced via Stark mixing are the usual rules.

If $S_{D E}\left(S_{D M}\right)$ is the electric (magnetic) line strength of the $J_{1} \rightarrow J_{2}$ line and $c$ is the speed of light in vacuum, the dimensionless quantity $\alpha$ $=c^{-1} \sqrt{S_{D M} / S_{D E}}$ compares the strength of the electric interaction with the strength of the magnetic interaction. For typical atomic values, $\alpha$ is half of the finestructure constant. This indicates a predominance of electric dipolar interactions. For lanthanide-doped crystals, the electric line strength of a transition inside the $4 f$ subshell of the lanthanide ions induced via the crystal field is often much weaker than the typical values of the free atoms. Therefore $\alpha \sim 1$ is reachable. In this case, the magnetic dipolar interaction competes with the electric dipolar interaction. If a transition is both dipolar electric and dipolar magnetic allowed, $\alpha$ is also the square root of the ratio of the polarizability and the magnetizability. Therefore, in order to realize left-handed materials, it is impor- 
tant to obtain $\alpha \sim 1$ as similar values of the polarizability and the magnetizability are required.

The remainder of this Letter focuses on erbium ions. It is well known that the magnetic interaction plays a significant role in the transition between the ground multiplet $J=15 / 2$ and the first excited multiplet $J=13 / 2$ of erbium ions ${ }^{10}(\alpha=0.69)$. This transition occurs for an electromagnetic radiation oscillating at approximately $1.54 \mu \mathrm{m}$ (depending on the host lattice).

The lower levels $m= \pm 1 / 2$ of the $J=15 / 2$ multiplet and the $J=13 / 2$ multiplet and the transition probabilities are displayed in Fig. 1. If the electric and the magnetic transitions occur exactly at the same frequency, the permittivity and the permeability cannot have both negative real parts (see the dashed curves in Fig. 2). A strategy to slightly shift the two transition frequencies is to apply a static magnetic field $\boldsymbol{B}_{0}$ along the quantization axis $\boldsymbol{z}$, defined by the host lattice. In this case, the remaining degeneracy $m$ $= \pm 1 / 2$ is removed by the Zeeman effect as the $\sigma_{+}$and $\sigma_{-}$transition frequencies are shifted from the $\pi$ transition frequency. As the Landé factors of the two multiplets are quite similar $\left(g_{15 / 2} \simeq 1.2\right.$ and $\left.g_{13 / 2} \simeq 1.1\right)$, the splitting of the $\pi$ transitions is much smaller than the damping rate of the quantum coherence and can be neglected. If the magnetic field induces the $\pi$ transition and the electric field induces the $\sigma_{+}$and $\sigma_{-}$ transitions, the permittivity and the permeability both can have a negative real part. This can be done for a probe electromagnetic radiation propagating along the $\boldsymbol{x}$ axis with an electric field linearly polarized along the $\boldsymbol{y}$ axis. For this configuration, the erbium-doped crystal presents a left-handed domain of frequency as shown on Fig. 2. The detuning $\delta$ is defined as the difference between the probe frequency and the frequency of the transition without the Zeeman effect.

The interaction of the probe field with the medium has been analyzed in the framework of the semiclassical theory. The steady states of the quantum coherence were determined up to first order in the interaction Hamiltonian. This assumes the field to be weak enough to neglect population transfers. In the calculations we use a density of the erbium ions of 5 $\times 10^{26} \mathrm{ions} / \mathrm{m}^{3}$ and a static magnetic field of $7.1 \mathrm{mT}$.

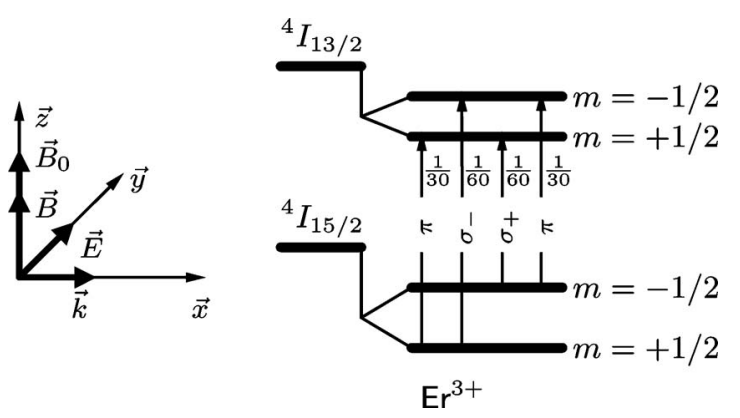

Fig. 1. Field polarization and energy levels of an erbium ion embedded in a host crystal. The probe field polarization dependence on the electronic transitions is schematically indicated, as are the corresponding transition probabilities. The energy levels are shifted by the Zeeman effect due to a static magnetic field $\boldsymbol{B}_{0}$.

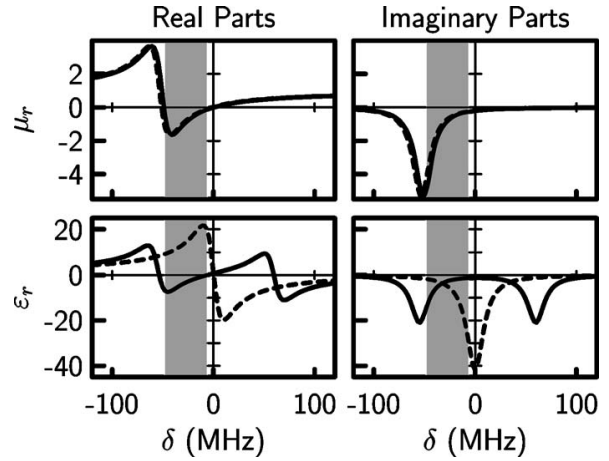

Fig. 2. Dependence of the permittivity and the permeability on the detuning. The erbium-doped crystal displays lefthanded properties in a bandwidth of approximately $50 \mathrm{MHz}$ (shaded area). In the case of a vanishing static magnetic field (dotted curve), no left-handed domain is found.

The damping rates of the quantum coherences are $10 \mathrm{MHz}$. This value is significantly bigger than the population decay rates (approximately $100 \mathrm{~Hz}$ ) and should be reached between liquid helium and liquid nitrogen temperatures. Lower values of the quantum coherence damping rates enhance the response of the medium but restrict the left-handed spectral domain.

It is worth noting that this result is not restricted to erbium ions. They have been chosen for the wellknown magnetic character of the $J=15 / 2 \rightarrow J=13 / 2$ transition and for the technological importance of the transition frequency. Nevertheless, many transitions in various lanthanide ions are known to possess a magnetic character, such as the transitions ${ }^{4} I_{9 / 2}$ $\rightarrow{ }^{2} I_{11 / 2}$ in $\mathrm{Nd}^{3+}$ at approximately $5 \mu \mathrm{m}(\alpha \simeq 0.2),{ }^{5} I_{8}$ $\rightarrow{ }^{3} K_{8}$ in $\mathrm{Ho}^{3+}$ at approximately $470 \mathrm{~nm}(\alpha \simeq 0.1)$, and ${ }^{4} I_{13 / 2} \rightarrow{ }^{2} K_{15 / 2}$ in $\mathrm{Er}^{3+}$ at approximately $360 \mathrm{~nm}(\alpha$ $\simeq 0.3$ ), for example. ${ }^{10}$

In conclusion, left-handed properties of lanthanidedoped crystals have been demonstrated in a finite domain of parameters. The result is based on the analysis of the electronic structure of rare earth ions embedded in a host lattice. Explicit calculations made for erbium ions predict a left-handed spectral domain of $50 \mathrm{MHz}$ for a probe field oscillating at approximately $1.54 \mu \mathrm{m}$.

The authors acknowledge the support of the Fonds National de la Recherche Scientifique (Belgium) and the Interuniversity Attraction Pole ProgramBelgian Science Policy. Q. Thommen's e-mail address is quentin.thommen@phlam.univ-lille1.fr.

\section{References}

1. V. G. Veselago, Usp. Fiz. Nauk 92, 517 (1964); [Sov. Phys. Usp. 10, 509 (1968)].

2. J. B. Pendry, Phys. Rev. Lett. 85, 3966 (2000).

3. R. A. Shelby, D. R. Smith, and S. Schultz, Science 292, 77 (2001).

4. V. M. Shalaev, W. Cai, U. K. Chettiar, H. Yuan, A. K. Sarychev, V. P. Drachev, and A. V. Kildishev, Opt. Lett. 30, 3356 (2005).

5. M. Ö. Oktel and Ö. E. Müstecaplioğlu, Phys. Rev. A 70, 053806 (2004). 
6. Q. Thommen and P. Mandel, Phys. Rev. Lett. 96, 053601 (2006).

7. J. D. Jackson, Classical Electrodynamics, 2nd ed. (Wiley, 1975).

8. H. A. Bethe, Ann. Phys. 3, 133 (1929); [Selected Works of Hans A. Bethe With Commentary (World Scientific, 1996), Vol. 8].

9. B. R. Judd, Phys. Rev. 127, 750 (1962).

10. A. A. Kaminskii, Laser Crystals, Springer Series in Optical Science (Springer, 1997). 